\title{
The Flow Field Analysis and Structure Optimization of Large Diameter Butterfly Valve
}

\author{
Wang Yuhao ${ }^{1,}$, He Qingzhong ${ }^{2}$ \\ ${ }^{1}$ Aerospace Science and Technology Microsystem Technology Co., Ltd, Nanjing, China \\ ${ }^{2}$ College of Mechanical Engineering, Sichuan University of Science and Engineering, Zigong, China
}

Email address:

15196007990@163.com (Wang Yuhao)

${ }^{*}$ Corresponding author

To cite this article:

Wang Yuhao, He Qingzhong. The Flow Field Analysis and Structure Optimization of Large Diameter Butterfly Valve. American Journal of Mechanical and Industrial Engineering. Vol. 5, No. 4, 2020, pp. 59-63. doi: 10.11648/j.ajmie.20200504.12

Received: August 24, 2020; Accepted: September 11, 2020; Published: September 21, 2020

\begin{abstract}
The large diameter butterfly valve used in Qinghai Naqu hydropower station was subjected to strong vibration when the opening was small. In view of the phenomenon of strong vibration and damage of the valve, the fluid theory analysis is carried out and the appropriate mathematical model of fluid analysis is selected. This paper established the flow model of butterfly valve for different opening degrees with the 2D drawings and simulated the flow field by using CFX software. Through numerical simulation, the flow field characteristics of the valve under different opening degrees are obtained, and it is found that the valve failure is caused by vortex street effect. The results of numerical analysis show that the vortex are the most strong in the range of $5 \%$ to $30 \%$ and The vortex street phenomenon is the strongest at $5 \%$ opening. When the opening is greater than $30 \%$, the vortex street effect decreases gradually. Meanwhile, the reason of vortex street effect is that the structure of butterfly plate is complex, and an improved structure was given and the results of numerical analysis show that the vortex effect decreased significantly for the new one. This new structure can effectively prevent the vortex resonance and give an example for the new design of a large butterfly valve.
\end{abstract}

Keywords: Butterfly Valve, CFX Software, Vortex Resonance, Structure Optimization

\section{Introduction}

As one of the most advanced butterfly valves, triple eccentric butterfly valve has the best sealing performance and is widely used in petroleum, chemical and electric power industries. At present, in the central and western regions of China, the main control valve of medium and large hydropower stations generally uses large diameter butterfly valve or large ball valve. However, butterfly valve has certain advantages over ball valve in manufacturing, controllability and sealing, so the research on large butterfly valve is of great significance [1].

For large diameter butterfly valve, it is difficult to obtain accurate test data of large butterfly valve due to test conditions and cost. Referring to the relevant literature, most of the design of large diameter butterfly valve in China is mainly based on traditional experience, which leads to the increase of production cost. In this paper, the flow field characteristics of the main control triple eccentric butterfly valve with the diameter of $3400 \mathrm{~mm}$ in gaqu hydropower station of Qinghai Province as the carrier, aiming at the instability of the system under small opening in practical application, the flow field characteristics are analyzed and studied.

Referring to the relevant literature, there are few researches on the flow analysis of three eccentric valves, especially the large-scale ones. The research in related fields mainly depends on theoretical empirical formula calculation. At present, the vibration phenomenon of valve is mainly focused on the mechanical and electrical system of valve opening and closing control, and the research on valve fluid characteristics is less. In this paper, the flow field analysis of butterfly valve with different opening degrees is carried out, and the vortex flow field near the butterfly plate is found. The related achievements are innovative, and there is no relevant research on large-scale triple eccentric butterfly valve at home and abroad. 


\section{Vortex Street Resonance}

When the viscous incompressible fluid flows around the body or through the orifice, the fluid will fall off the body and form a nonlinear periodic

Line vortex flow, namely vortex street phenomenon. Vortex street phenomenon is a typical fluid phenomenon in nature, such as when the fluid bypasses the high-rise buildings, electric poles and heat exchange tubes in the heat exchanger, vortex street phenomenon will occur [2].

The prevention and elimination of vortex street resonance is very important for the structure design and opening and closing system design of butterfly valve. Large hydropower main control butterfly valve often works in small opening state in the off-season of water resources. As the butterfly valve is a combined welding part, the structure of butterfly plate is relatively complex, which will lead to strong eddy current in the negative pressure area of butterfly plate when water flows through the butterfly plate at small opening. When the vortex street frequency is equal to or close to the natural frequency of butterfly valve, resonance will occur [3], which will bring great damage to the valve system Bad.

At present, the vortex street resonance phenomenon of butterfly valve in small opening can not be accurately predicted, and the traditional design method can only rely on empirical formula to do qualitative analysis. In this paper, the flow field of butterfly valve is numerically simulated based on finite element analysis method, the distribution law of vortex street phenomenon is found, and the structure of butterfly valve is optimized.

\section{Theoretical Basis of Turbulence Model for CFX Flow Field Analysis}

In the theory of hydrodynamics, turbulence is a kind of flow with complex flow pattern and poor controllability. Radshaw et al. Pointed out that the subtle density change does not have obvious effect on the flow. Here, ignoring the effect of density fluctuation, assuming that the fluid inside the butterfly valve is an incompressible viscous fluid (i.e., the density is constant), the governing equation of turbulent average flow is obtained as follows [4]:

Equation of continuity:

$$
\frac{\partial \rho}{\partial t}+\frac{\partial}{\partial x_{i}}\left(\rho u_{i}\right)=0
$$

momentum equation (Navier-Stokes):

$$
\frac{\partial}{\partial t}\left(\rho u_{i}\right)+\frac{\partial}{\partial x_{i}}\left(\rho u_{i} u_{j}\right)=-\frac{\partial \rho}{\partial x_{i}}+\frac{\partial}{\partial x_{j}}\left(\mu \frac{\partial u_{i}}{\partial x_{j}}-\rho \overline{u_{i} u_{j}}\right)+S_{i}
$$

The above two equations are the basic turbulence equations, $\rho$ represent the density of fluid, $u_{i}, u_{j}$ represent the components of velocity vector in $\mathrm{X}$ and $\mathrm{Y}$ directions, $\mu$ represent the viscosity of fluid, $S$ represent the source term.
The Navier Stokes equation and the theory of velocity boundary layer are commonly used to study the vortex street phenomenon in fluid flow. In this paper, the flow in the upper part of the butterfly valve is equivalent to the flow around the edge of the butterfly plate in the case of small opening. According to the three-dimensional fluid model, the $k-\omega$ equation is used to solve the $N-S$ equation. That is, the characteristics of the $k-\omega$ model are retained on the wall of the butterfly plate, and the $k-\mathcal{E}$ model is used far away from the wall surface, and this is the most suitable turbulence to simulate vortex shedding phenomenon Flow model [5].

$k$ equation:

$$
\frac{\partial}{\partial_{t}}(\rho k)+\frac{\partial}{\partial x_{i}}\left(\rho k u_{i}\right)=\frac{\partial}{\partial x_{j}}\left(\Gamma_{k} \frac{\partial k}{\partial x_{j}}\right)+P_{k}-Y_{k}
$$

$\omega$ equation:

$$
\frac{\partial}{\partial t}(\rho \omega)+\frac{\partial}{\partial x_{i}}\left(\rho \omega u_{i}\right)=\frac{\partial}{\partial x_{j}}\left(\Gamma_{\omega} \frac{\partial \omega}{\partial x_{j}}\right)+P_{\omega}-Y_{\omega}+D_{\omega}
$$

$k$ represent turbulent kinetic energy, $\omega$ represent Diffusivity, $P_{k}$ is the generating term of $k, P_{\omega}$ is the generating term of $z, Y_{k}$ is the dissipation term of $k, Y_{\omega}$ is the dissipation term of $\omega, D_{\omega}$ is the Orthogonal diffusion term, $\Gamma_{k}$ is the effective diffusion coefficient of $k, \Gamma_{\omega}$ is the effective diffusion coefficient of $\omega$.

\section{Numerical Simulation of Flow Field}

\subsection{Establishment of Flow Field Mathematical Model}

Firstly, the three-dimensional mathematical model of butterfly valve is established by SolidWorks, and the three-dimensional model of butterfly plate is shown in Figure 1. (a). Due to the complex structure of butterfly valve, this paper introduces the established three-dimensional model into ABAQUS software [6], and obtains the accurate mathematical model of flow channel by Boolean subtraction operation, as shown in Figure 1. (b).

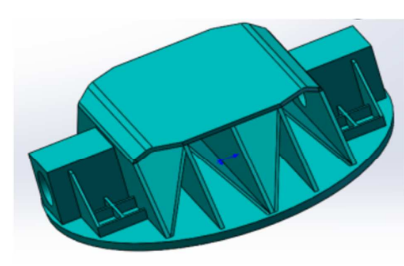

(a)

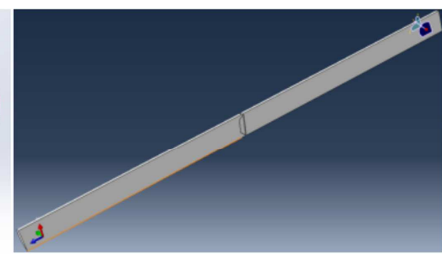

(b)
Figure 1. (a) $3 D$ model of butterfly plate; (b) Channel model at $10 \%$ opening.

Figure 1. (b). shows the mathematical model of the flow field when the opening is $10 \%$. In order to be consistent with the actual working state of the butterfly valve as much as possible, and to ensure that the fluid medium entering the 
butterfly valve and the final output butterfly valve is in a stable flow state, in the analysis and calculation of the flow field characteristics, the input and output pipes with a length of 10 times the nozzle diameter are added at the inlet and outlet ends of the butterfly valve [7]. Because of the symmetry of the fluid in the butterfly valve, the half model is used to reduce the amount of calculation.

\subsection{CFX Numerical Simulation}

In this paper, with the help of the workbench platform in ANSYS to analyze the flow field [8], the mesh module is used to mesh the channel model, and the mesh is locally encrypted. Finally, 54716 meshes and 11940 nodes are obtained, as shown in Figure 2. (a) [9].

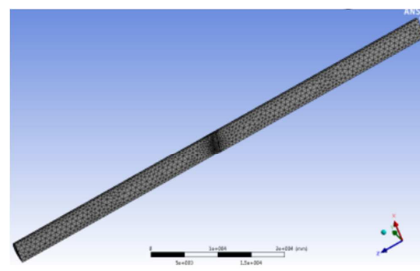

(a)

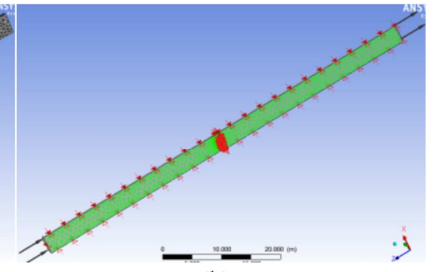

(b)

Figure 2. (a) Grid model;(b) Boundary condition

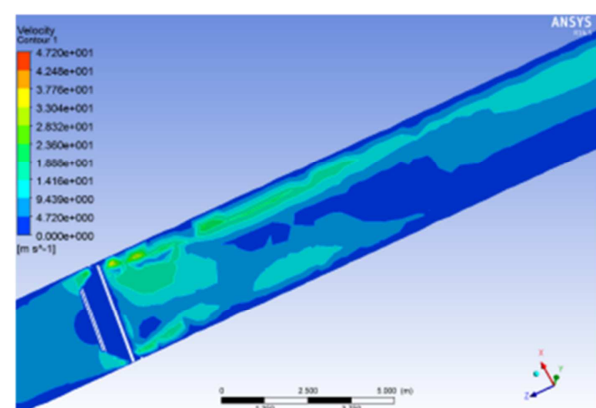

(a) Velocity distribution of symmetry plane at $10 \%$ opening

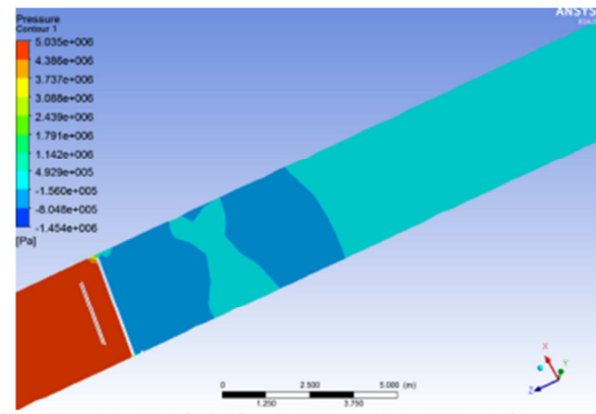

(c) Pressure distribution on symmetry plane at $10 \%$ opening

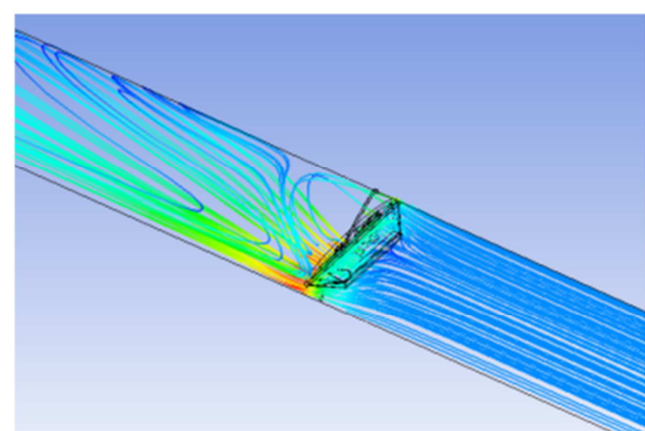

(e) Streamline diagram under $10 \%$ opening
Since the analysis is a half model, symmetry constraint is imposed on the symmetry plane of the model. The actual inlet and outlet boundary conditions of butterfly valve are: inlet pressure $5 \mathrm{MPa}$, outlet pressure $0.15 \mathrm{Mpa}$, as shown in Figure 2 . (b). The fluid medium is water at 25 degrees, the analysis condition is the steady flow analysis of incompressible fluid, and the turbulence model is selected. The calculated residual diagram is shown in Figure 3.

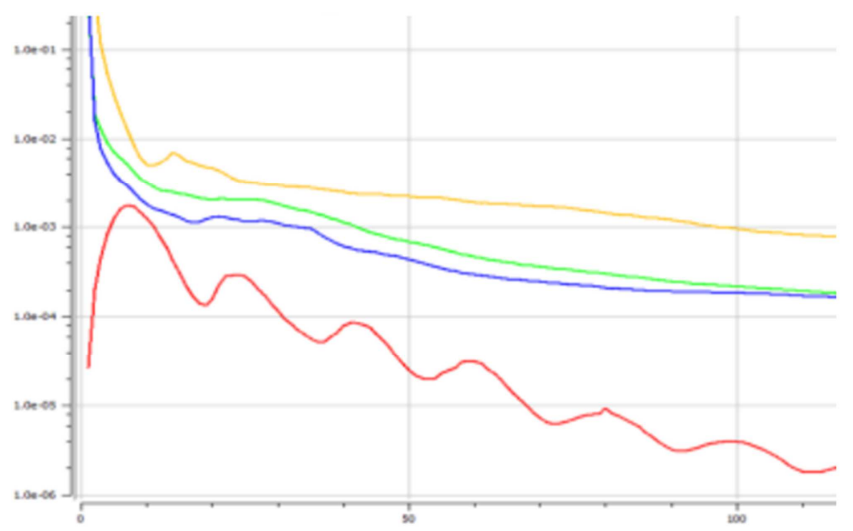

Figure 3. Residual curve.

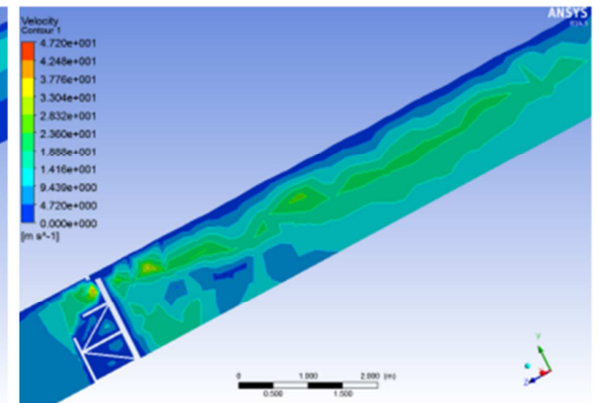

(b) Velocity distribution of center plane at $10 \%$ opening

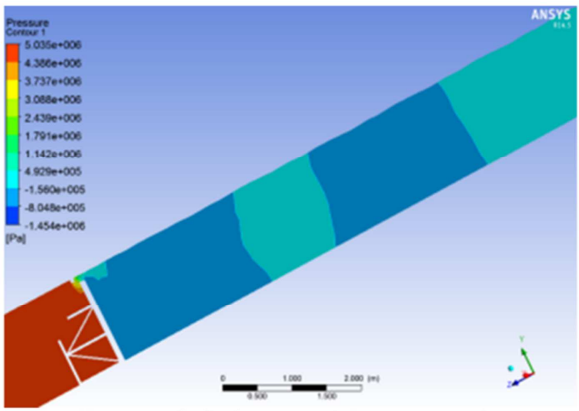

(d) Pressure distribution on central plane at $10 \%$ opening

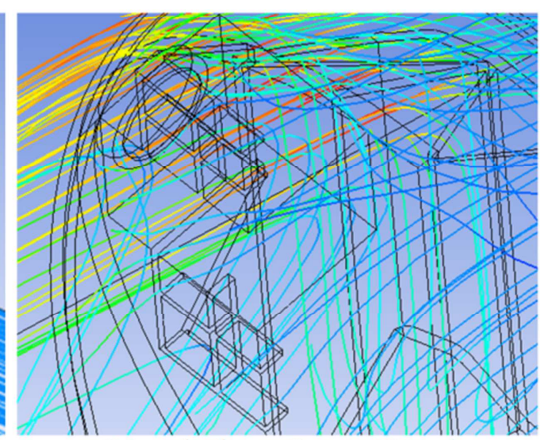

(f) Streamline diagram of journal at $10 \%$ opening

Figure 4. Result nephogram. 


\section{Calculation Results and Analysis}

\subsection{Simulation Results}

In this paper, the flow field of the valve under the conditions of $5 \%, 10 \%, 20 \%, 30 \%, 40 \%, 50 \%, 60 \%, 70 \%, 80 \%, 90 \%$, $100 \%$ opening was analyzed, and the corresponding flow field characteristics were obtained. Due to many analysis results, this paper only presents the velocity distribution and pressure distribution under the condition of $10 \%$ opening, as shown in Figure 4 , in which the symmetry plane is the interface of the half model, and the central plane is the plane passing through the center of the valve stem.

\subsection{Result Analysis}

When the opening is small, the gap between the edge of butterfly plate and the sealing ring of valve seat is very small, and the velocity of fluid increases and the pressure decreases,

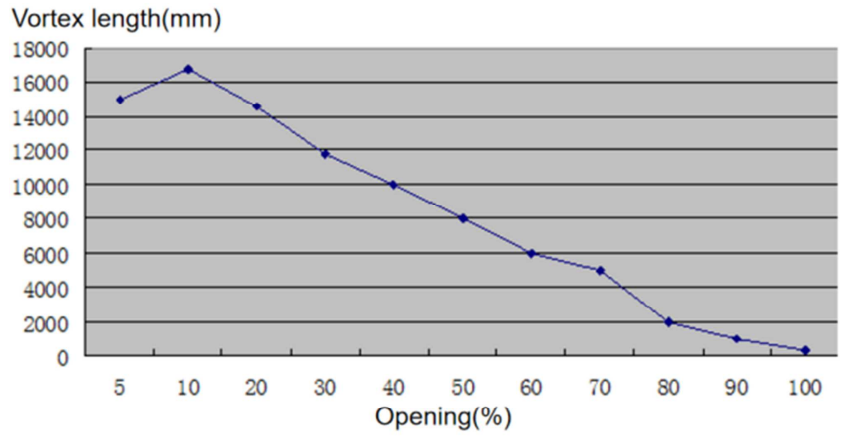

(a) resulting in throat effect. The throat effect causes strong eddy current at the front and back of the butterfly plate edge, as shown in Figure 4 (a), (b). Due to the complex structure of the butterfly plate journal and many rib plates, the flow around the butterfly plate and the journal is disordered and the flow direction changes rapidly, as shown in Figure 4 (e), (f). When the fluid flows around the edge of the butterfly plate, a vortex street flow is formed downstream of the valve, and the fluid tends to be stable at the position of 5 times the pipe diameter (about $17000 \mathrm{~mm}$ away from the back of the butterfly plate). Through the fluid simulation with different opening degrees, the change law of vortex street phenomenon and the velocity characteristics of vortex area are obtained, as shown in Figure 5: (in Figure 5. (a), the vortex length in the ordinate indicates that the vortex street appears with the back of butterfly plate as the zero point Area length of the image)

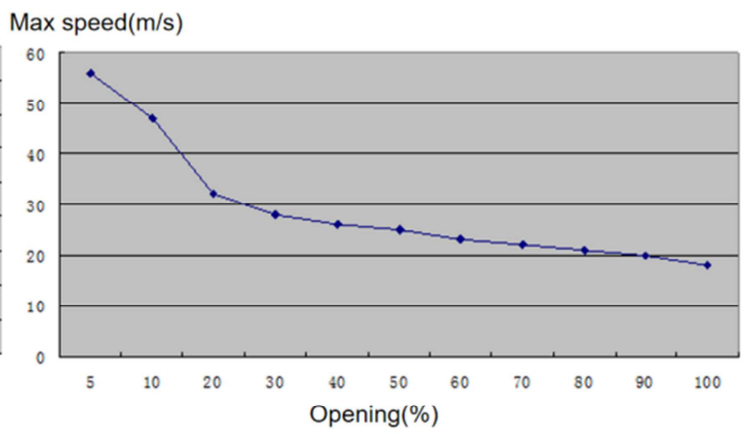

(b)

Figure 5. (a) Influence area of vortex street under different opening;(b) Maximum eddy current velocity at different opening.

It can be seen from Figure 5. that the length of vortex area decreases linearly with the increase of opening. The maximum vortex velocity decreases sharply in the range of 5\%-20\%, and tends to be stable after $30 \%$ opening. The results show that the vortex street phenomenon downstream of the valve is strong when the opening is small, and the vortex street effect gradually weakens with the increase of the opening. This is consistent with the strong vibration of the valve in the small opening state [10].

The large diameter hydropower butterfly valve is different from the small diameter butterfly valve. Its butterfly plate is a welded assembly. As shown in Figure 5. (a), there are many structural rib plates, which leads to the relatively complex flow of water through the butterfly valve.

\subsection{Comparative Analysis Before and After Optimization}

The main influence factor of butterfly valve flow field is the structure of butterfly plate. From the previous numerical simulation results, it can be seen that the structure mutation at the Journal of butterfly valve is large and there are many rib plates, which leads to the rapid change of fluid flow direction at the upstream of butterfly valve; the vortex street effect of butterfly plate originates from the gap between the sealing edge of butterfly plate and the valve seat when the opening is small, and the edge angle of butterfly plate will lead to the flow direction To mutation, so as to create conditions for the generation of vortex street [11]. The sealing edge of butterfly plate is rounded with R10, and the square of upper and lower journal is made into semicircle, and the rib plate near the journal is improved. The improved structure is shown in Figure 6. Using the same analysis method, the improved analysis results are obtained. The comparative statistics of the data before and after the improvement are shown in Table 1, Table 2.

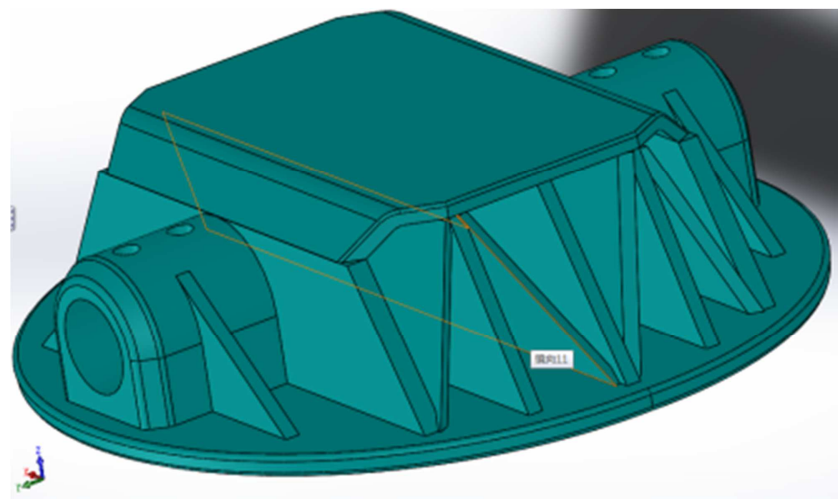

Figure 6. Improved butterfly plate. 
Table 1. Comparison before and after optimization (from 5\% to 50\%).

\begin{tabular}{llllllll}
\hline Opening & & $\mathbf{5 \%}$ & $\mathbf{1 0 \%}$ & $\mathbf{2 0 \%}$ & $\mathbf{3 0 \%}$ & $\mathbf{4 0 \%}$ & $\mathbf{5 0 \%}$ \\
\hline \multirow{3}{*}{ Vortex length (mm) } & Before optimization & 15 & 16.8 & 14.6 & 11.8 & 10 & 8 \\
& After optimization & 9.4 & 9.8 & 9.35 & 8.1 & 8 & 7.8 \\
& Difference & 5.6 & 7 & 5.25 & 3.7 & 2 & 0.2 \\
\multirow{3}{*}{ Max eddy velocity (m/ s) } & Before optimization & 56 & 47 & 32 & 28 & 26 & 25 \\
& After optimization & 41 & 30 & 23 & 25 & 24 & 22 \\
& Difference & 15 & 17 & 9 & 3 & 2 & 3 \\
\hline
\end{tabular}

Table 2. Comparison before and after optimization (from 60\% to 100\%).

\begin{tabular}{lllllll}
\hline Opening & & $\mathbf{6 0 \%}$ & $\mathbf{7 0 \%}$ & $\mathbf{8 0 \%}$ & $\mathbf{9 0 \%}$ & $\mathbf{1 0 0}$ \\
\hline \multirow{3}{*}{ Vortex length $(\mathrm{m})$} & Before optimization & 6 & 5 & 2 & 1 & 0.3 \\
& After optimization & 5.9 & 4.95 & 1.97 & 0.98 & 0.29 \\
& Difference & 0.1 & 0.05 & 0.03 & 0.02 & 0.01 \\
\multirow{3}{*}{ Max eddy velocity (m/ s) } & Before optimization & 23 & 22 & 21 & 20 & 18 \\
& After optimization & 22 & 21 & 20 & 20 & 19 \\
& Difference & 1 & 1 & 1 & 0 & 1 \\
\hline
\end{tabular}

After the improvement, there is no sudden change in structure and the fluid flow is more gentle than before. It can be seen from table 1 that after structural improvement, the maximum reduction of eddy current influence area is $7000 \mathrm{~mm}$ and the maximum eddy current velocity is reduced by $17 \mathrm{~m} / \mathrm{s}$. Among them, the eddy current weakens obviously in the range of 5\%-30\%, and the influence of eddy current has little change after $30 \%$ opening. Through the structural improvement, the eddy current effect at low opening is effectively reduced, which is beneficial to avoid resonance phenomenon [12], it provides help for similar valve design in the future.

\section{Conclusion}

This paper analyzes the flow field of large diameter butterfly valve of a hydropower station, and improves the structure of butterfly plate, the following conclusions can be drawn:

(1) Through the three-dimensional modeling software to establish the three-dimensional model, using ABAQUS software topology calculation to the complex flow channel model, and finally relying on the workbench platform of ANSYS to simulate the flow field of large diameter butterfly valve, which provides an effective method for the subsequent similar valve design.

(2) Through the analysis and calculation, the flow field characteristics of butterfly valve under different opening degrees are obtained. The results show that the vortex street phenomenon of large diameter butterfly valve is strong under small opening, which is consistent with the strongest vibration of valve under small opening in practical engineering.

(3) Through the improvement of butterfly plate structure, the vortex street phenomenon in the flow field area of butterfly valve is obviously weakened, which plays a positive role in preventing resonance, and provides ideas for the optimization design of valve in the future.

\section{References}

[1] TONG Chengbiao. Optimization design and flow field research of butterfly valve. Changsha, School of mechanical and transport engineering, Hunan University, 2010.

[2] LIli, SHI Zhihui, ZAHO Jinglong. Numerical simulation of flow field around vortex street and vortex flowmeter. Journal of Dalian Jiaotong University, 2014, 35 (6): 32-35.

[3] DENG Jian, LI Jinghu, ZHU Ayuan. Research on Karman vortex street effect of UAV with skid. Aircraft design, 2014, 34 (3): 1-3.

[4] Min Qiangli. Numerical simulation of Karman vortex street with low Reynolds number. Journal of Sichuan military engineering, 2009, 30 (11): 81-83.

[5] Pang Lijun, LV Guiping, Zhong Su. Vortex street simulation and vibration analysis of turbine fixed guide vane. Journal of mechanical engineering, 2011, 47 (22): 159-166.

[6] Shi Yiping, Zhou Yurong. ABAQUS finite element analysis example details. Beijing: China Machine Press, 2006: 126.

[7] Zhang Qinzhao, Liu Fusheng, Wang Hong. Study on flow field and resistance characteristics of triple eccentric butterfly valve. Fluid machinery, 2013, 41 (11): 1-5.

[8] Ding Yuan, Wu Jihua. ANSYS CFX 14.0 from introduction to mastery. Beijing: Tsinghua University Press, 2013.

[9] Jia Rumin, Zhang Weizheng. CFD numerical simulation and flow path optimization of control valve. Chemical machinery, 2009, 36 (2): 131-134.

[10] Li Jianwei, Xiao Liangyu. Research on vibration characteristics of butterfly valve disc of Francis turbine. Valve, 2013, 6: 15-17.

[11] Wang Dong, Zhang Siqing and Zhang Yunlong. Dynamic analysis and dovetail modification of turbine fixed guide vane vortex street. China electric power, 2014, 0 (11): 5-9.

[12] Li Tong, Zhou Jianjia, Yuan Shouqi. Static analysis of spiral centrifugal pump impeller based on flow field calculation. Fluid machinery, 2013, 41 (12): 22-26. 